\title{
An Amplitude Modulation of Cerebral Rhythms based Method in a Motor Task BCI Paradigm
}

\author{
Oana Diana Eva, Anca Mihaela Lazar \\ Medical Bioengineering Faculty \\ "Grigore T. Popa" University of Medicine and Pharmacy \\ Iasi, Romania
}

\begin{abstract}
Quantitative evaluation based on amplitude modulation analysis of electroencephalographic signals is proposed for a brain computer interface paradigm. The method allows characterization of the interaction effects of different frequency bands in the electroencephalographic rhythms during motor tasks. A new index was proposed and computed to be a measure of the amplitude modulation. Built on this index, features vector are established for training different classification algorithms. Signals recorded from 50 subjects revealed important differences in amplitude modulations between motor tasks. Most notably, Theta modulation of the Theta and Alpha rhythms proved to be reliable discriminant features between different mental tasks.
\end{abstract}

Keywords-Brain computer interface; motor tasks; electroencephalographic signal; amplitude modulation analysis; classifiers

\section{INTRODUCTION}

Brain computer interface (BCI) provides a new communication channel for people with motor disabilities using electroencephalographic signals (EEG) or other brain signals.

EEG is often used in BCI systems because it is not an invasive experiment, has no risk and has good time resolution.

The oscillatory activity in the EEG is classified according to rhythms: Delta (0.1-4 Hz), Theta $(4-8 \mathrm{~Hz})$, Alpha $(8-12 \mathrm{~Hz})$, Beta $(12-30 \mathrm{~Hz})$, and Gamma $(30-60 \mathrm{~Hz})$.

Motor imagery (MI) is a dynamic state during which a given action is mentally simulated by a subject [1]. Sensory stimulation, motor behavior, movement or only imagination of movement can modify the functional links in the cortex and cause an amplitude decrease in the EEG, called Event Related Desynchronization (ERD), or an increase in the amplitude of the signal, named Event Related Synchronization (ERS) of the $\mathrm{Mu}$ or Beta rhythm. The Mu rhythm is a normal central rhythm in Alpha frequency band. Mu waves appear in the sensorimotor (SM) cortex.

Different feature extraction methods are reported for discriminating the motor tasks in a BCI paradigm: spectral analysis [2], autoregressive methods [3], independent component analysis [4], [5], Itakura distance [4] and phase synchronization [4], [6].

The main objectives of the proposed method are to discriminate, extract and classify representative EEG features which can be used in brain computer interface motor imagery paradigm by means of an amplitude modulation analysis.

Studies conducted so far using amplitude modulation analysis have shown that there are differences between the EEG from healthy subjects and EEG from subjects diagnosed with Alzheimer's disease [7], [8]. In our case, the differences that appear during motor imagery tasks (left hand imagination, right hand imagination and relaxation) are studied.

It is researched the possibility of using a quantitative method of analyzing the EEG signal. Amplitude modulation analysis is computed for EEG signal rhythms and then some classification methods are applied.

Section II describes the dataset used, the methodology that includes the proposed method and the classification methods. The results are presented in section III, the discussions in section IV and the conclusions in section V.

\section{MATERIALS AND METHOD}

\section{A. Dataset and Subjects}

The database is formed by 50 EEG signals collected from 50 healthy volunteers, men and women, age range 19-59 years, untrained. The EEG signals were recorded with g.MOBIlab+ portable acquisition system provided by g.tec Guger Technologies together with the BCI2000 platform. Eight electrodes (CP3, CP4, P3, P4, C3, C4, PZ and CZ) were placed on the volunteers scalp according to International 10-20 System. The reference electrode was linked on the right earlobe. Experimental protocol consisted of randomly right or left motor tasks and relaxation periods. The volunteers were comfortably seated on a seat in front of a PC monitor that displays left or right arrows. They must look carefully at the arrows and try to imagine the hand movement indicated by the arrow. When the screen is white, the subject needs to relax. Each arrow appears for 30 times. The time interval between visual stimuli was 2 seconds and the sampling frequency used was $256 \mathrm{~Hz}$. Before the experiment, the subject is instructed not to speak, to move or to blink during the trials. The recordings took place on different days, under brightness conditions chosen by the volunteers. All volunteers provided written informed consent prior to the experiment.

\section{B. Data Processing}

Three datasets were created associated to the motor imagery tasks: one representing the EEG during the left hand 
imagination, one representing EEG during the right hand imagination and one representing EEG during the relaxation.

The EEG signal $x(n)$ is decomposed into three frequency bands (cerebral rhythms): $4-8 \mathrm{~Hz}$ (Theta rhythm), $8-12 \mathrm{~Hz}$ (Alpha rhythm), $12-30 \mathrm{~Hz}$ (Beta rhythm).

$x_{i}(n)=x(n) * \mathrm{~h}_{i}(n)$,

Where $\mathrm{h}_{i}(n), \mathrm{i}=1,2,3$ represents the impulse response of the applied bandpass filter corresponding to each frequency band. We justify later why there are only three rhythms used.

The Hilbert transform $H\{\cdot\}$ of $x_{i}(n)$ signal is:

$\mathcal{H}\left\{x_{i}(n)\right\}=\frac{1}{\pi} P V \int_{-\infty}^{+\infty} \frac{x_{i}(\tau)}{t-\tau} d t$,

where $P V$ is the Cauchy principal value.

The analytical signal is denoted by:

$\overline{x_{l}(n)}=x_{i}(n)+j \mathcal{H}\left\{x_{i}(n)\right\}$.

The amplitude modulation $e_{i}(n)$ of each cerebral rhythm is the modulus of $x_{i}(n)$ [7], [8]:

$e_{i}(n)=\sqrt{x_{i}(n)^{2}+\mathcal{H}\left\{x_{i}(n)\right\}^{2}}$.

The $e_{i}(n)$ is multiplied by a $5 \mathrm{~s}$ Hamming window which is shifted across the data set with a step of $0.5 \mathrm{~s}$. The amplitude modulation for frame $m$ is represented as $e_{i}(m, n)$, where $n$ is the time variable.

For each rhythm $i$ the absolute value of the Fourier transform is computed for each sub band $j$.

$E_{i}(m ; f)=\left|\mathcal{F}\left\{e_{i}(m, n)\right\}\right|$,

where $\mathcal{F}\left\{e_{i}(m, n)\right\}$ is the discrete Fourier transform of the temporal envelope for each frame $e_{i}(m, n)$ and $f$ denotes modulation frequency.

In order to quantify the rate of change of the temporal envelope and the potential frequency interactions, the modulation frequencies are subsequently arranged to coincide with the first four conventional frequency ranges from Delta to Beta.

Gamma band modulation frequencies $(30-60 \mathrm{~Hz})$ can only be present for gamma rhythm. Therefore, for this reason, but also to reduce the amount of data, the modulation band corresponding to gamma rhythm is not taken into account in this type of analysis. The Delta rhythm is not taken into consideration because there is only one situation (the modulation band corresponding to the Delta rhythm consisting of the lowest frequencies $0.1-4 \mathrm{~Hz}$ ). Therefore, we work with three rhythms and four modulation bands, which lead to nine options. Assigning the band modulation_rhythm notation (e. g. Delta_Theta represents modulation in Delta band of the Theta rhythm), all the possibilities are depicted in Table 1.

With the aim of quantifying the weight of the different modulation frequencies in the cerebral rhythms, a new parameter called Modulation_index is proposed:

$\left(\text { Modulation_index }{ }_{m}\right)_{i, j}=\frac{\overline{E_{l, j}(m)}}{\sum_{i=1}^{3} \sum_{j=1}^{4} \overline{E_{l, j}(m)}}$
TABLE I. MODULATION BANDS CORRESPONDING TO THE CEREBRAL RHYTHMS

\begin{tabular}{|l|l|l|l|l|}
\hline \multirow{2}{*}{ Rhythm } & \multicolumn{4}{|l|}{ Modulation Band } \\
\cline { 2 - 5 } & Delta & Theta & Alpha & Beta \\
\hline Beta & Delta_Beta & Theta_Beta & Alpha_Beta & Beta_Beta \\
\hline Theta & Delta_Theta & Theta_Theta & Alpha_Theta & --------------- \\
\hline Alpha & Delta_Alpha & Theta_Alpha & --------------- & --------------- \\
\hline
\end{tabular}

where $\overline{E_{l, J}(m)}$ is the average on all frames (resulting from the segmentation of the EEG signal with the Hamming slidingwindow) of the Fourier transform moduli corresponding to the modulation frequency band $j$ of the rhythm $i$, and $\sum_{i=1}^{3} \sum_{j=1}^{4} \overline{E_{l, j}(m)}$ is the sum of all Fourier transform module averages for all rhythms and for all possible modulation bands.

\section{Features Extraction, Selection and Data Classification}

Discriminations between the left and the right motor activity (left-right), the left motor activity and relaxation (LeftRelaxation), the right motor activity and relaxation (RightRelaxation) are evaluated with five classifiers: linear discriminant analysis (LDA) [9], quadratic discriminant analysis (QDA) [10], Mahalanobis distance (MD) [11], k nearest neighbor $(\mathrm{kNN})$ [12] and support vector machine (SVM) [13]. A 10x10 fold cross validation method estimate the classification rates.

The aims of the research concern in finding the modulation band/s related to a specific cerebral rhythm and the classifier/s which best discriminate between the classes (left - right, leftrelaxation or right - relaxation).

\section{RESULTS}

The implementation of the proposed method is in MATLAB.

There are already mentioned the three cases under study, namely left-right, left-relaxation and right-relaxation.

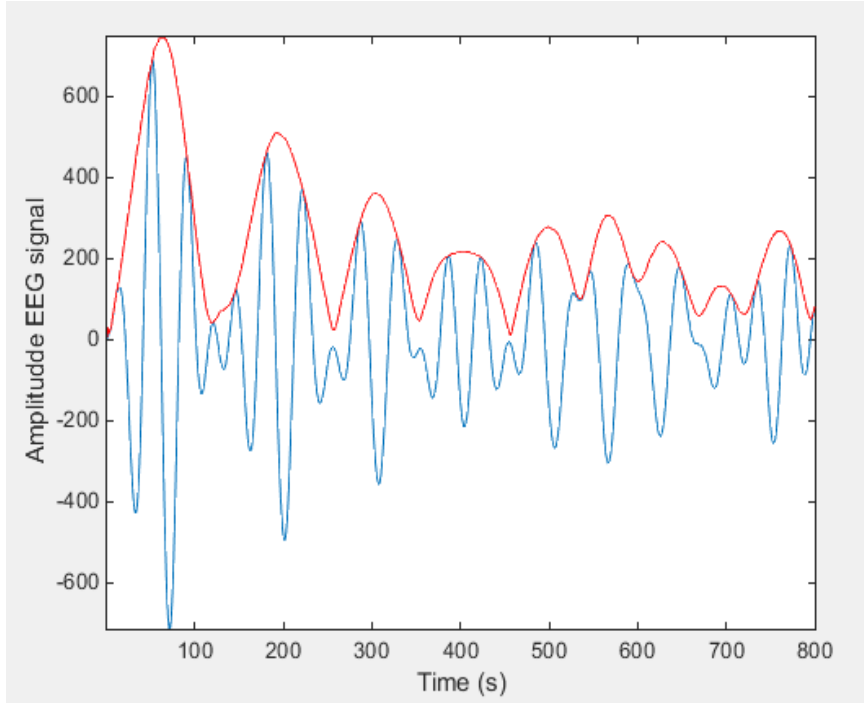

Fig. 1. EEG Alpha Rhythm from Channel C3 (blue) and Theta Amplitude Modulation (red) for Subject SL27i when he Imagines the left Hand Movement. 


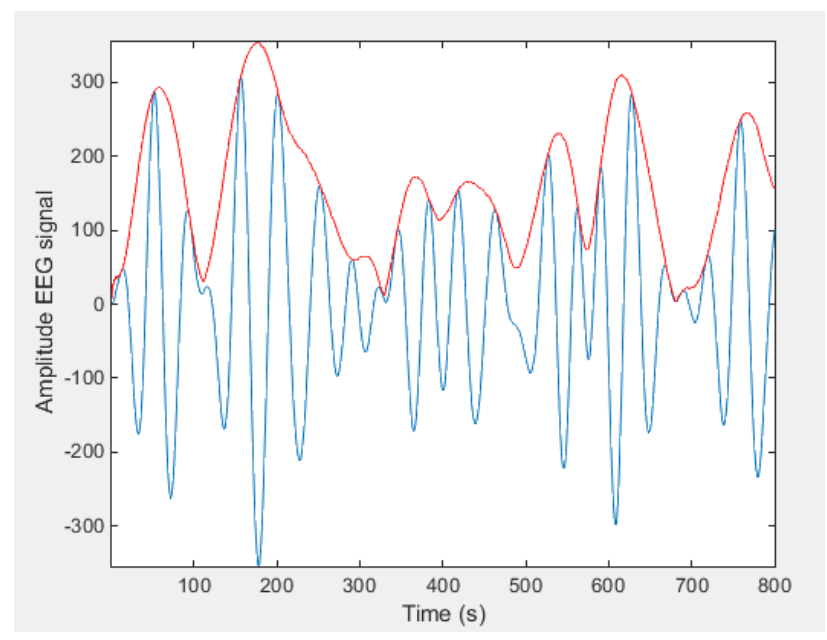

Fig. 2. EEG Theta Rhythm from Channel C4 (blue) and Delta Amplitude Modulation (Red) for Subject SL27i when he Imagines the left Hand Movement.

For each case, the recorded EEG signals are IIR bandpass filtered, on the corresponding frequency bands of the three rhythms under study (Theta, Alpha and Beta).

Then using (2), (3) and (4) the amplitude modulations are computed. The plots of Fig. 1 and Fig. 2 exemplify an EEG rhythm in blue and one of its envelopes in red.

For each case, the proposed new indexes, computed by means of (5) and (6) for the corresponding two classes, generate the consistent feature vector. For each subject from of all the 50 subjects the maximum of the classification rates estimated by all the mentioned classifiers is further extracted (for each rhythm and each corresponding modulation bands). So we get 50 maxima for each situation. Hereafter, there are taken into account only those values of maxima higher or equal to a high threshold considered to be $80 \%$. The results are presented in Table II.

A result from the first cell from Table II is interpreted in the following manner: in the left-right case, there are 19 subjects from the all of 50 subjects whose classification rates are higher or equal to $80 \%$.

TABLE II. THE NUMBER OF THE MAXIMA OF THE CLASSIFICATION RATES HIGHER OR EQUAL TO $80 \%$

\begin{tabular}{|c|c|c|c|}
\hline Modulation_Rhythm & left-right & left-relaxation & right-relaxation \\
\hline Delta_Theta & 19 & 19 & 21 \\
\hline Theta_Theta & 20 & 19 & 19 \\
\hline Delta_Alpha & 8 & 20 & 14 \\
\hline Theta_Alpha & 8 & 20 & 23 \\
\hline Alpha_Alpha & 9 & 14 & 19 \\
\hline Delta_Beta & 8 & 12 & 7 \\
\hline Theta_Beta & 14 & 13 & 16 \\
\hline Alpha_Beta & 9 & 6 & 16 \\
\hline Beta Beta & 3 & 8 & 10 \\
\hline
\end{tabular}

The green cells from Table II draw attention to the situations when the number of classification rates higher or equal to $80 \%$ exceeds a proper (high enough) value, considered by us being equal to 19 . It is obvious that many cases are related to Theta rhythm, both for Delta and Theta modulation. There are no noteworthy differences for the three cases reported (left-right, left-relaxation and right-relaxation). Also, in the Alpha rhythm and modulation in Theta and Alpha bands, in the right-relaxation case, there are a significant number of classification rates greater than $80 \%$. So, for Theta_Alpha, the classification rates are above $80 \%$ for almost a half of the subjects (23 from 50 subjects). In the left-relaxation case, high values are obtained for Theta and Delta modulation bands. It is worth noticing that low values are achieved for left-right (8 or 9 from 50 subjects). So we conclude that in left-right paradigm the Alpha rhythm modulations are presented in few persons, so we ignore it.

In order to evaluate the results attained for each classifier, there are considered only those green marked situations in Table II.

The results for left-right case are illustrated in Fig. 3 where only the Theta rhythm is under study.

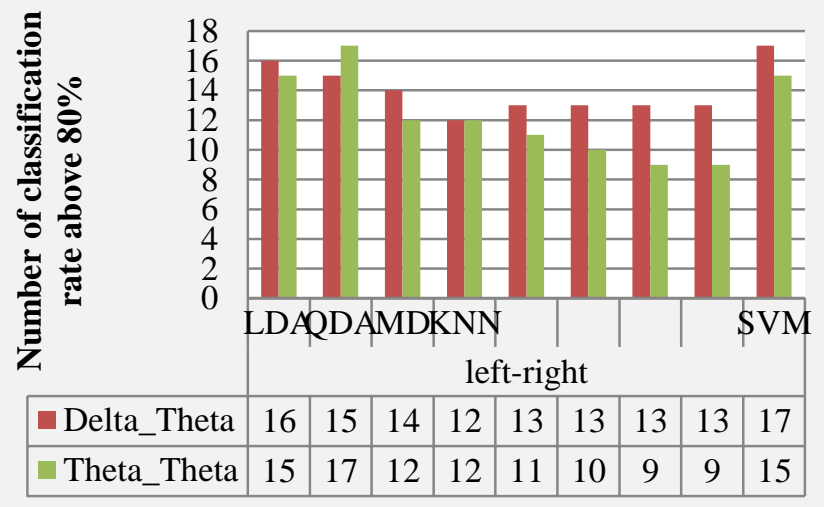

Fig. 3. Number of Classification Rates Higher than $80 \%$, for all the Classifiers in the Left-Right Case

It is easy to observe that the best results are achieved by means of LDA, QDA and SVM classifiers.

The results for left-relaxation are gathered in Fig. 4. The modulations of Theta and Alpha rhythms are considered.

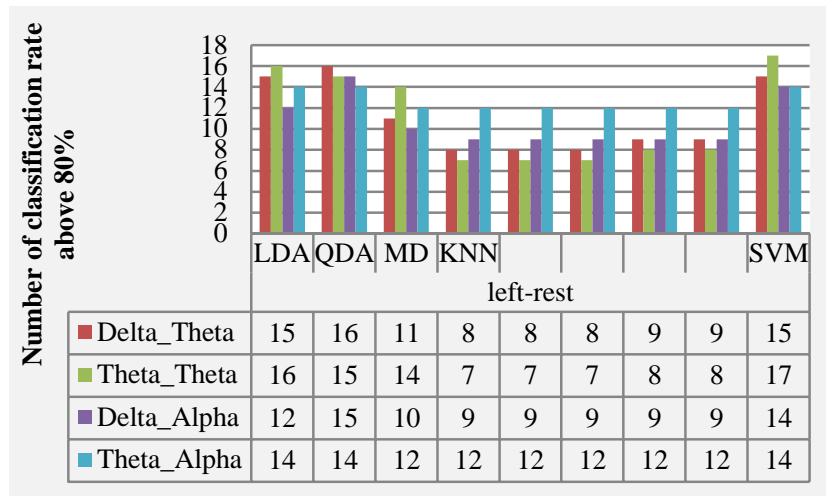

Fig. 4. Number of Classification Rates Higher than $80 \%$, for all the Classifiers in the Left-Relaxation Case. 
LDA, QDA and SVM are the best classifiers also in the case presented in Fig. 4.

The results for right-relaxation are drawn together in Fig. 5. As in the previous case, the modulations of Theta and Alpha rhythms are taken into consideration.

As in the two cases, the best results are found for LDA, QDA and SVM classifiers.

For further considerations, we have to mention that for Theta_Theta, in left-right case, there are 7 classification rates upper $90 \%$, and one with $100 \%$.

As for all the cases, LDA, QDA and SVM classifiers lead to high classification rates, in order to look deeper into and to study the distributional characteristics, the box plots are used.

Fig. 6 illustrates the box plots for LDA classifier for the left-right case, Left-relaxation and Right-relaxation cases (Delta_Theta and Theta_Theta).

In Fig. 7 the box plots for LDA classifier for the left-right case, left-relaxation and right-relaxation cases (Delta_Alpha Theta_Alpha and Alpha_Alpha) are presented.

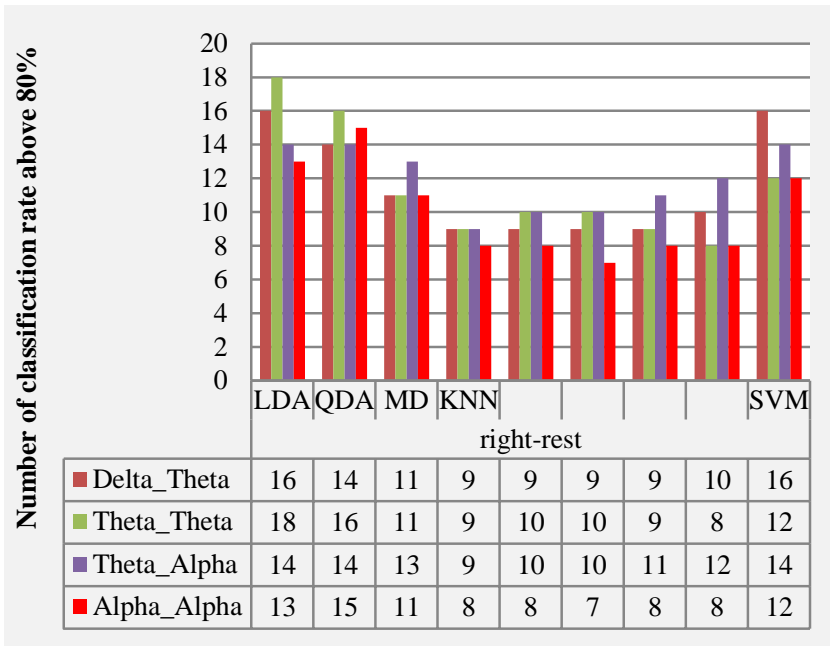

Fig. 5. Number of Classification Rates Higher than $80 \%$, for all the Classifiers in the Right-Relaxation Case.

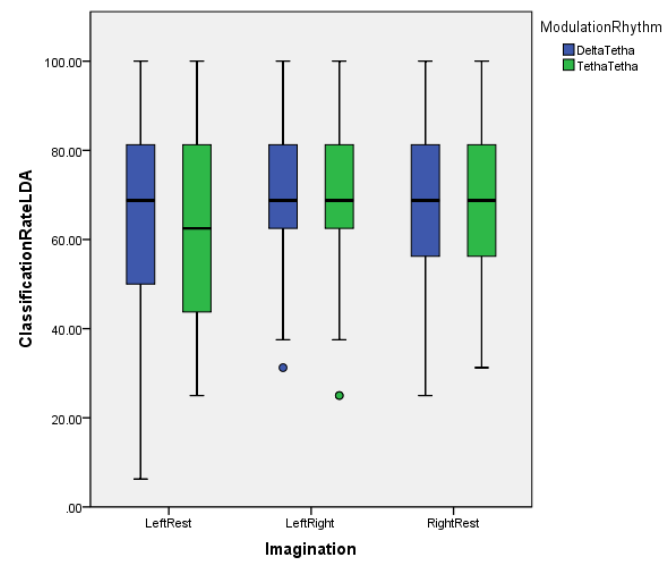

Fig. 6. The Box Plots for LDA Classifier for the Left-Right Case, LeftRelaxation and Right-Relaxation Cases (Delta_Theta and Theta_Theta).

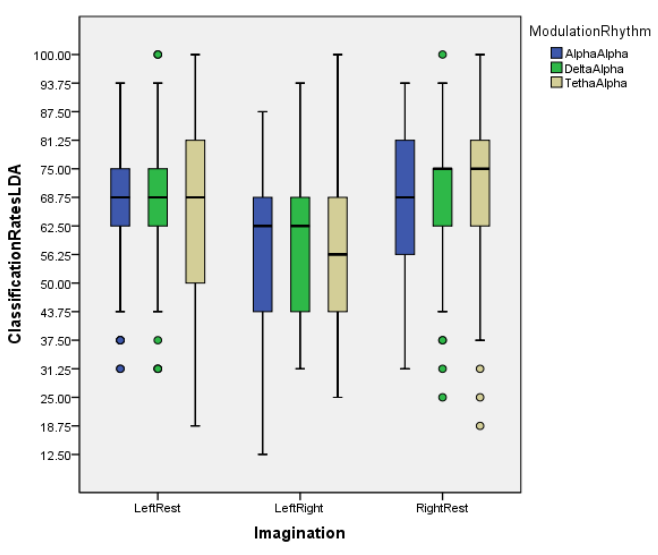

Fig. 7. The Box Plots for LDA Classifier for the Left-Right Case, LeftRelaxation and Right-Relaxation Cases (Delta_Alpha Theta_Alpha and Alpha_Alpha).

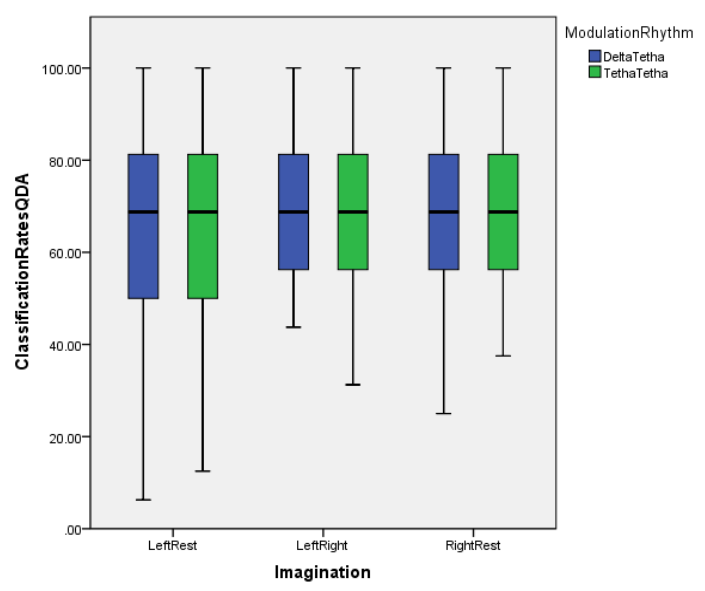

Fig. 8. The Box Plots for QDA Classifier for the Left-Right Case, LeftRelaxation and Right-Relaxation Cases (Delta_Theta and Theta_Theta).

Fig. 8 shows the box plots for QDA classifier for the Leftright case, left-relaxation and right-relaxation cases (Delta_Theta and Theta_Theta).

In Fig. 9 the box plots for QDA classifier for the left-right case, left-relaxation and right-relaxation cases (Delta_Alpha Theta_Alpha and Alpha_Alpha) are displayed.

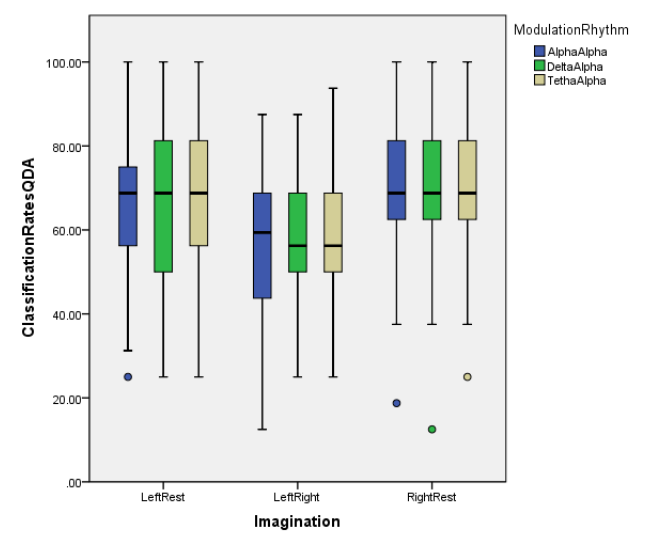

Fig. 9. The Box Plots for QDA Classifier for the Left-Right Case, LeftRelaxation and Right-Relaxation Cases (Delta_Alpha Theta_Alpha and Alpha_Alpha). 


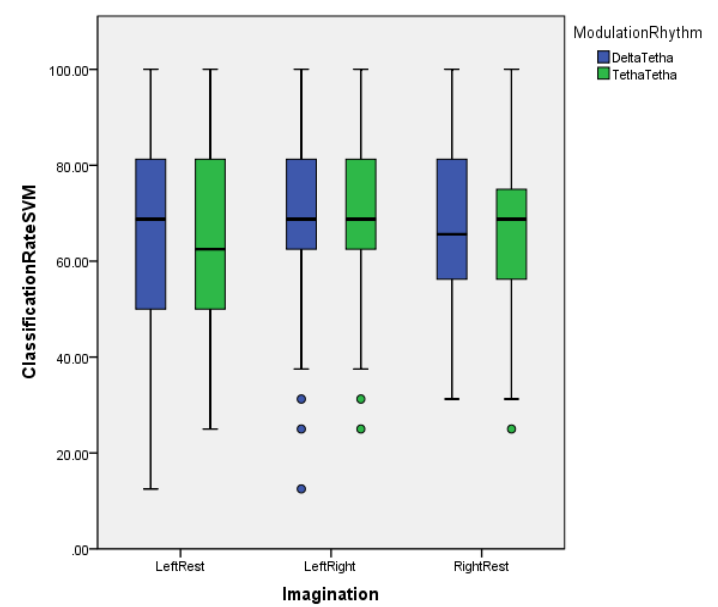

Fig. 10. The Box Plots for SVM Classifier for the Left-Right Case, LeftRelaxation and Right-Relaxation Cases (Delta_Theta and Theta_Theta).

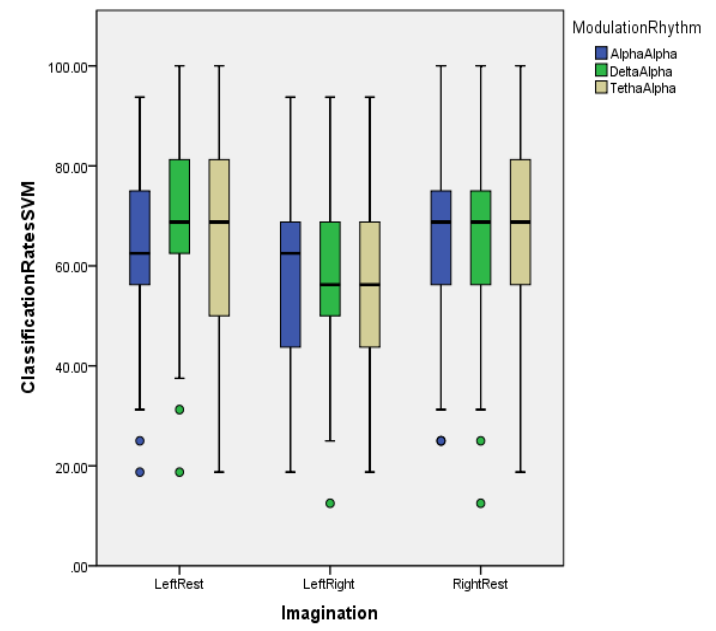

Fig. 11. The Box Plots for SVM Classifier for the Left-Right Case, LeftRelaxation and Right-Relaxation Cases (Delta_Alpha Theta_Alpha and Alpha_Alpha).

Fig. 10 and Fig. 11 indicate the box plots for SVM classifier for the left-right case, left-relaxation and rightrelaxation cases (Delta_Theta and Theta_Theta) and (Delta_Alpha Theta_Alpha and Alpha_Alpha) respectively.

When the midpoint and spread of the classification rates are examined, we can observe that the lowest medians (lower than $60 \%$ ) are for all the classifiers, for the left-right case in Alpha rhythm, for all amplitude modulation bands (Delta, Theta and Alpha). The medians are about $70 \%$ in almost all the other situations. The spread is evaluated by the interquartile range box (which represents the middle $50 \%$ of the data) and by the whiskers. Good results from this point of view are attained both for LDA and QDA classifiers, for right-relaxation situation, in Theta rhythm modulated by Theta band. Here the interquartile range is between $58 \%$ and $80 \%$ classification rate and there are no outliers. For LDA, even better results are attained for left-right case for Theta_Theta or Delta_Theta when the interquartile range is between $63 \%$ and $80 \%$, but there are two outliers. For all the situations where good results are reported, the whiskers extend to $100 \%$.

\section{DISCUSSIONS}

We have to mention that it is impossible to make direct comparisons to other results reported on public EEG databases, because herein our own database has been handled. In a future work, we intend to validate the amplitude modulation analysis on the available public databases, especially as the EEG signals are recorded on more than 8 channels and better outcomes might be attained.

But comparisons must be done with related works. So, to assess the benefits of the proposed method by means of the amplitude modulation metric (Modulation_index) we do compare our findings with the results from [4] and [12], validated on the same database.

In [4], where Itakura distance based method, phase synchronization methods and independent component analysis are used, the classification rates are approximately the same with the actual results and most of classification rates greater than $80 \%$ are obtained with QDA, kNN and SVM. Phase synchronization metric conducted to the lowest classification rates (about 60\%) and symmetric Itakura distance to the highest ones (about 90\%).

By means of wavelet (Coiflet 4 and Daubenchies 2) coefficients and kNN classifiers, in [12], the results were between $68 \%$ and $91 \%$ (only one subject).

So, the herein results ( 7 classification rates better than $90 \%$ and one value of 100\%) outperform those from [4], [12].

In order to improve the rate of classification for all the subjects, we will try to propose a further index/parameter which would better characterize the neuromodulatory activity of the brain, maybe to combine with other features and to employ also other classifiers such as Deep Neural Network (DNN) [14].

It is worth to mention that it is important to have trained subjects. So, we will develop a new database with many sessions of recordings.

\section{CONCLUSIONS}

The method highlights the frequency bands the cerebral rhythms in a motor task based paradigm are modulated with and provides additional information over conventional methods.

EEG amplitude modulation analysis has revealed the best result concerning classification rate (discriminative patterns) between imagination of right hand movement and relaxation period when the Theta rhythm is modulated with Theta band using LDA and QDA classifiers and between imagination of right and left hand movement when the Theta rhythm is modulated with Theta or Delta bands, working with LDA classifier.

The results showed that EEG activity both for Theta and Alpha rhythms are modulated by the Theta band in a different manner when a person imagine the right hand movement, or left hand movement or he/she relax.

We can conclude that the proposed method is helpful in separating motor tasks for a BCI paradigm. 


\section{REFERENCES}

[1] R. Carabalona, P. Castiglioni P, F. Gramatica, "Brain-computer interfaces and neurorehabilitation", Stud Health Technol Inform., 145:160-76, 2009.

[2] J.R. Wolpaw, N. Birbaumer, D.J. McFarland, G. Pfurtscheller, T.M. Vaughan, "Brain computer interfaces for communication and control", Clinical Neurophysiology, 113(6), pp. 767-791, 2002.

[3] D.J. McFarland, J.R. Wolpaw, "Sensorimotor rhythm-based braincomputer interface (BCI): model order selection for autoregressive spectral analysis", Journal of neural engineering, 5(2), 155, 2008.

[4] O. D. Eva, A. M. Lazar, Feature Extraction and Classification Methods for a Motor Task Brain Computer Interface: A Comparative Evaluation for Two Databases, (IJACSA) International Journal of Advanced Computer Science and Applications, Vol. 8, No.8, 2017.

[5] A. Hyvärinen, "Independent component analysis: recent advances", Phil Trans. R. Soc. A, 371(1984), p.20110534, 2013.

[6] V. Gonuguntla, Y. Wang, KC Veluvolu, "Phase synchrony in subjectspecific reactive band of EEG for classification of motor imagery tasks", InEngineering in Medicine and Biology Society (EMBC), pp. 27842787, 2013

[7] TH Falk, FJ Fraga, L. Trambaiolli, R. Anghinah, "EEG amplitude modulation analysis for semi-automated diagnosis of Alzheimer's disease", EURASIP Journal on Advances in Signal Processing, 1(192), 2012 .
[8] FJ Fraga, TH Falk, PA Kanda, R. Anghinah, "Characterizing Alzheimer's disease severity via resting-awake EEG amplitude modulation analysis", PLoS One, 8(8):e72240, 2013.

[9] B. Blankertz, S. Lemm, M. Treder, S. Haufe, K.R. Müller, "Single-trial analysis and classification of ERP components - a tutorial", NeuroImage, 56(2), 814-825, 2011.

[10] F. Lotte, M. Congedo, A. Lécuyer, F. Lamarche, B. Arnaldi, "A review of classification algorithms for EEG-based brain-computer interfaces" J. Neural Eng., 4, pp. R1-R13, 2007.

[11] A. Bashashati, M. Fatourechi, R.K. Ward, G.E. Birch, "A survey of signal processing algorithms in brain-computer interfaces based on electrical brain signals", Journal of Neural engineering, 4(2), R32, 2007.

[12] R. Aldea, M. Fira, A. Lazăr, "Classifications of motor imagery tasks using k-nearest neighbors", InNeural Network Applications in Electrical Engineering (NEUREL), IEEE, pp. 115-120, 2014.

[13] SM Zhou, JQ Gan, F. Sepulveda, "Classifying mental tasks based on features of higher-order statistics from EEG signals in brain-computer interface", Information Sciences, 178(6):1629-40, 2008.

[14] Abeer Al-Nafjan, Manar Hosny, Areej Al-Wabil, Yousef Al-Ohali, "Classification of Human Emotions from Electroencephalogram (EEG) Signal using Deep Neural Network", (IJACSA) International Journal of Advanced Computer Science and Applications, Vol. 8, No. 9, 2017. 